\title{
Pérdida de pubescencia foliar y sus efectos fisiológicos en Espeletia paipana (Asterales, Asteraceae), en el departamento de Boyacá-Colombia
}

\author{
Sindy Buitrago ${ }^{1}$, Leidy Vanegas ${ }^{1} \&$ Carolina Ramos $^{2}$ \\ 1. Grupo de Manejo Biológico de Cultivos, Escuela de Ciencias Biológicas, Universidad Pedagógica y Tecnológica de \\ Colombia, Av. Central Norte 39-115 Tunja, Colombia; biopao.k18@gmail.com, leidyvanegas_8915@hotmail.com \\ 2. Grupo de investigación en Manejo Integrado de Ecosistemas y Biodiversidad. Escuela de Ciencias Biológicas, \\ Universidad Pedagógica y Tecnológica de Colombia, Av. Central Norte 39-115 Tunja, Colombia; \\ carolina.ramos@uptc.edu.co
}

Recibido 17-IX-2014. Corregido 11-II-2015. Aceptado 17-III-2015.

\begin{abstract}
Loss of leaf pubescence and its physiological effects on Espeletia paipana (Asterales, Asteraceae), in the Boyacá-Colombia department. Espeletia paipana is an endangered giant caulescent rosette endemic to Boyacá department. In order to establish whether a plant disease, characterized by the loss of leaf pubescence (PPF) and attributed to the pathogenic action of endophytic microorganisms, is the cause of the increasing mortality of population, the physiological performance of the species was evaluated with and without PPF. The incidence (\% leaves affected in each of the 27 individuals in the current population) and severity (\% leaf area affected on 135 leaves) of the PPF were monitored over a period of nine months, in three topographic zones of different heights. During four consecutive days in both dry and wet season, physiological parameters as chlorophyll content index (ICC), stomatal conductance (Gs) and leaf temperature (Tfol) were measured in healthy and affected leaves. The study was complemented with isolations and pathogenicity tests to identify the causal agent of the PPF. Overall, although the disease incidence in E. paipana was constant over time, the severity progressed surpassing $60 \%$ of the leaf area. The increasing of severity in the upper side of leaves was attributed to the photo-oxidative effect of high radiation between 11:00 h and 14:00 $\mathrm{h}$ of the day. The reduction of functional leaf area because of the PPF, led to low Gs with serious implications for carbon fixation and thus limiting growth and biomass renewal. The effect of season in Tfol varied according to the topographic zone, while the ICC did not present a defined pattern with respect to the PPF; its low values could be associated with the production of other pigments. Finally, although it is not possible to ensure that Botrytis sp. is the causative of the loss of leaf pubescence, it is postulated as the most probably causal agent due to its high representativeness in the isolates and its infectious potential during the pathogenicity tests. In general, the reduction of healthy leaf biomass and decrease of physiological performance suggest that PPF affect negatively the survival of E. paipana, which means that the use of biological controllers could be a strategy to mitigate its effect on the population. Rev. Biol. Trop. 63 (3): 845-858. Epub 2015 September 01.
\end{abstract}

Key words: Espeletia paipana, pubescence, pathogenic disease, severity, leaf temperature, stomatal conductance, chlorophyll content.

Los páramos son ecosistemas tropicales de alta montaña que se caracterizan por presentar condiciones drásticas a causa de la alta radiación, baja temperatura anual, frecuentes heladas nocturnas y alta humedad relativa (Monasterio, 1980; Hofstede, 1995; Castrillo, 2006). La alternancia de irradiación directa durante el día y congelamiento y gelifluxión durante la noche genera amplias variaciones de temperatura que superan los regímenes estacionales (Monasterio, \& Sarmiento, 1991; Luteyn, 1992). En general, estas condiciones abióticas conllevan a que los páramos tengan una baja productividad primaria, una lenta descomposición de la biomasa, difícil absorción de nutrientes y retraso de los procesos sucesionales (Luteyn, 1999; Sánchez, 2003).

Colombia tiene una extensión de 14400 $\mathrm{km}^{2}$ de páramo, que aunque corresponden a más de un $40 \%$ de la extensión mundial, en 
realidad sólo representan el $1.3 \%$ del territorio Colombiano (Hofstede, Segarra, \& Mena, 2003). Diversas actividades como la ganadería, agricultura, quema, deforestación y extracción de recursos biológicos son actualmente fuertes factores antrópicos que llevan a que el territorio de páramo sea cada vez más reducido, afectando la sobrevivencia de especies de fauna y flora específicas para este ecosistema (Vargas, Jaimes, Castaño, \& Mora, 2004; Alonso-Amelot, 2008; Castaño, 2002).

Espeletia paipana S. Díaz \& Pedraza es una roseta caulescente endémica del departamento de Boyacá, que se encuentra en la categoría En Peligro Crítico por la UICN, debido a su dramática reducción poblacional, ocupando un área menor a $0.007 \mathrm{~km}^{2}$ (López, 2004). Para el año 2004 se reportó un total de 76 individuos entre adultos, jóvenes y plántulas, de los cuales, actualmente sobreviven 27 individuos en su mayoría adultos.

Con base en observaciones detalladas de las hojas de E. paipana, en el 2012 detectamos una enfermedad foliar que se caracteriza por la pérdida de pubescencia, lesión que en estados avanzados causa la formación de manchas necróticas, clorosis y deformación de la hoja. El descubrimiento de la enfermedad dio origen al interrogante sobre si la acelerada mortandad poblacional estaba relacionada con la afectación por dos vías simultáneas: el estrés abiótico a causa de la disminución de la regulación hídrica y térmica que representa la pérdida de pubescencia foliar (PPF), y el estrés biótico, generado por la acción patogénica de microorganismos asociados a la lesión en el tejido fotosintético.

La pubescencia foliar es una característica de gran valor adaptativo para las plantas del páramo, debido a que atenúa los efectos negativos de condiciones ambientales adversas sobre el estado fisiológico y el crecimiento. Es una modificación de la epidermis que varía tanto en longitud como en densidad de acuerdo a la altitud, con el fin de regular la alta radicación incidente, la economía hídrica y la difusión de gases (Meinzer \& Goldstein, 1985, Molina-Montenegro, 2008). En espeletias, la pubescencia aumenta con el gradiente altitudinal permitiendo el desacople con la temperatura ambiente, es decir, actúa como regulador térmico que permite mantener los óptimos de temperatura para la fotosíntesis. Llega a reflectar más del $20 \%$ de la radiación incidente, reduciendo cerca de un $10 \%$ la cantidad de radiación asimilada por la hoja; aumenta el grosor de la capa límite, al disminuir la pérdida de calor por convección durante la transpiración, y retiene humedad, al reducir el efecto abrasivo y la desecación a causa del viento (Goldstein, Rada, Canales, \& Zabala, 1989; Azócar \& Rada, 1993; Levizou, Drilias, Psaras, \& Manetas, 2004; Castrillo, 2006).

Con relación a la infección foliar por patógenos, se sabe que la incidencia de fitopatógenos altera la susceptibilidad y/o tolerancia de las plantas a las condiciones del ambiente y al ataque de insectos (Paul, 1982; Fisher \& Petrini, 1991; Sempere \& Santamarina, 2008). Aspectos que posiblemente determinan en gran medida el éxito reproductivo y la permanencia de las poblaciones vegetales en el tiempo. Sin embargo, es escaso el conocimiento acerca del tipo de interacciones bióticas que rodean a la gran mayoría de especies vegetales de páramo y más aún, se desconoce el efecto conjunto del estrés biótico y abiótico sobre el desempeño fisiológico de las mismas.

En este estudio se evaluó el estado sanitario foliar y el rendimiento fisiológico de $E$. paipana con el fin de identificar los factores que han acelerado la reducción de su población. Este trabajo se llevó a cabo mediante el análisis del comportamiento de la incidencia y la severidad de la PPF, la caracterización de la comunidad de hongos asociados a esta patología y el ensayo de la capacidad infectiva de hongos altamente agresivos, aislados del tejido foliar afectado. Para determinar el efecto de la PPF en la fisiología de la especie se valoró la conductancia estomática, la temperatura foliar y el contenido de clorofilas. La identificación de una actividad patogénica y la alteración fisiológica asociada a la PPF, permitirá determinar estrategias de control y prevención para esta y otras espeletias. 


\section{MATERIALES Y MÉTODOS}

Área de estudio: El estudio se realizó durante el 2013 en el páramo La Cuchilla $\left(5^{\circ} 51^{\prime} 11.4^{\prime \prime} \mathrm{N}-73^{\circ} 0.8^{\prime} 33.7^{\prime \prime} \mathrm{W}\right)$ a una altitud entre los 3509 y 3567 m, dentro del Parque Natural Municipal Ranchería. El páramo es parte del Corredor Guantiva-La Rusia-Iguaque ubicado en el costado occidental de la Cordillera Oriental, en jurisdicción de los departamentos de Boyacá, Santander y Cundinamarca (Sánchez \& Cely, 2003; Solano, Roa, \& Calle, 2005). Este complejo presenta un régimen de lluvia bimodal comprendido entre Abril-Mayo y Octubre-Noviembre, y dos periodos secos en Diciembre-Marzo y Junio-Agosto con temperaturas promedio en época seca de $27.6{ }^{\circ} \mathrm{C}$ y en época húmeda de $25.7^{\circ} \mathrm{C}$. La precipitación anual fluctúa entre $700 \mathrm{~mm}$ y más de $3000 \mathrm{~mm}$ (Solano, et al., 2005).

La población vegetal del páramo La Cuchilla esta conformada por pastizales de Calamagrostis, matorrales de Hypericum, Chusquea y helechos del género Blechnum. E. paipana se encuentra asociada a poblaciones de E. nemekenei, E. incana, E. argentea y Espeletiopsis guacharaca (López, 2004).

Distribución de la población: La distribución de los 27 individuos de E. paipana en el sitio de estudio comprende tres zonas: cima (3567 m), pendiente $(3542 \mathrm{~m})$ y depresión (3509 m). La zona fue considerada como un factor determinante de las diferentes variables morfo-fisiológicas y sanitarias, razón por la cual se analizó su efecto en este estudio.

Dado que la condición más estresante en los páramos es la alta radiación lumínica, se midió la radiación fotosintéticamente activa (PAR) en el rango diurno de mayor intensidad solar, en intervalos de 15 minutos desde las 11:00 h hasta las 14:00 h usando un medidor Apogeé MQ-100.

Evaluación de la incidencia y la severidad: Se diagnosticó la pérdida de pubescencia foliar (PPF) tanto en el haz como en el envés de las hojas, como una enfermedad que conlleva la presencia de manchas necróticas, quemazón, clorosis y deformación. La incidencia de PPF se evaluó en cada uno de los 27 individuos como el porcentaje de hojas que presentaron los síntomas mencionados. Como un factor colateral a la PPF, se estimó la incidencia de la herbivoría por plagas (porcentaje de hojas con signos de esqueletización y defoliación). Los datos fueron tomados a los largo de nueve meses y analizados por zonas mediante el test de Kruskal Wallis. Además, se realizó una correlación mediante el coeficiente de Spearman para determinar el comportamiento de la herbivoría respecto a la PPF.

Por otro lado, la severidad se estimó como el porcentaje de área afectada de la hoja, calculado en cinco hojas por individuo seleccionadas al azar, fotografiadas y analizadas en el programa Axio Vision LE 4.8 (2009). Los datos de severidad fueron transformados con $\log _{10}$ $(\mathrm{x}+1) \mathrm{y}$ analizados mediante ANOVAS a una vía para identificar diferencias entre zonas, $\mathrm{y}$ determinar la variación de la severidad entre el haz y el envés de las hojas.

A partir de los resultados de incidencia y severidad se estimó el porcentaje de biomasa foliar con PPF, con base en el cual se clasificó a los individuos en tres niveles de afectación: bajo (0-5\%), medio (5-10\%) y alto (> $10 \%)$.

Mediciones morfo-fisiológicas: Durante cuatro días consecutivos en la época seca de enero y en la época húmeda de noviembre, se midió la conductancia estomática (Gs) y la temperatura foliar (Tfol) en dos hojas sanas y dos hojas con PPF de cada uno de los 27 individuos. Para mayor homogeneidad de los datos, los registros se realizaron durante las tres horas de mayor luminosidad simultáneamente a las mediciones de radiación PAR, utilizando un porómetro digital Decagon Devices SC-1. Así mismo, se midió el índice de contenido de clorofila (ICC): tres mediciones por hoja sana y tres mediciones en hojas afectadas, empleando un clorofilómetro OptiScience CCM-200.

Los datos fueron normalizados mediante la transformación tipo $\log _{10}$ para la Gs y el ICC y $\sqrt{ } \mathrm{x}$ para la variable Tfol, $\mathrm{y}$ analizados mediante 
ANOVA a dos vías, para observar el efecto de la PPF, la zona y la época. Las variables Gs y Tfol fueron correlacionadas con el coeficiente de Spearman para establecer cómo se relaciona el grado de enfriamiento foliar con la conductancia estomática.

Dentro de las características morfológicas, se midió el área foliar (Afol) y la altura de los individuos. El Afol se obtuvo del promedio de cinco hojas maduras por roseta mediante mediciones con regla de las dimensiones de la hoja, fotografías y su posterior análisis en el programa Axio Vision LE 4.8.

Aislamiento e identificación de hongos: Se obtuvieron muestras del tejido foliar afectado a partir de 15 hojas de E. paipana. Las muestras se almacenaron en bolsas sellables a $4{ }^{\circ} \mathrm{C}$ y se procesaron dentro de las 24 horas siguientes. El material se cortó en secciones pequeñas $\left(<1 \mathrm{~cm}^{2}\right)$ que comprendían el área afectada y parte del tejido sano colindante. La esterilización de los cortes se realizó por inmersión en alcohol $70 \%$ (1 min), en hipoclorito de sodio $1 \%$ (1 min) y cinco lavados de $1 \mathrm{~min}$ con agua destilada estéril de acuerdo con la metodología de French \& Hebert (1980). Posteriormente, se sembraron tres cortes en cajas de Petri utilizando Agar Papa Dextrosa (PDA), Agar malta y Agar nutriente. Las siembras se incubaron a $27^{\circ} \mathrm{C}$ durante ocho a 30 días. Los cultivos fueron purificados e identificados mediante microcultivos, empleando las claves taxonómicas de Barnett (1960), Hanlin (1990) y Castaño (1998).

Los resultados de los aislamientos se contrastaron con observaciones al microscopio de 20 improntas realizadas en campo. Para esto se tomaron muestras con cinta transparente que cubrieran el área afectada y sana de la hoja. Las muestras se colorearon con azul de metileno al colocar la cinta sobre la lámina portaobjetos.

Estudio de patogenicidad: Dado el estado de amenaza de E. paipana, es escaso el material foliar sano que puede extraerse de la zona de estudio. Para las pruebas de patogenicidad se realizaron tres pruebas de infección en hojas de E. paipana (4 hojas) y de E. nemekenei (20 hojas), empleando las cepas Botrytis sp., Fusarium sp. cepa F1 y Fusarium sp. cepa F2, aisladas del tejido foliar afectado. A partir del cultivo puro de cada cepa, se raspó el micelio superficialmente para obtener una suspensión de conidias en agua estéril y Tween al $0.5 \%$. La suspensión se observó al microscopio y se cuantificó mediante un hemocitómetro. Se preparó $5 \mathrm{~mL}$ de inóculo a una concentración de $10^{6}$ esporas $/ \mathrm{mL}$, que fue suministrada en dosis de $0.5 \mathrm{~mL}$ en el ápice, base y en el centro de la vena principal de la hoja empleando jeringas de $5 \mathrm{~mL}$.

Se evaluó el avance de la infección de cada cepa y la respuesta de un control (seis hojas sanas) cada dos a tres días durante un periodo de 42 días en el laboratorio, donde las hojas se mantuvieron en cámara húmeda bajo condiciones de luz difusa entre 18 a $68 \mu \mathrm{mol} / \mathrm{m}^{2}$.s, temperatura de $19{ }^{\circ} \mathrm{C}$ y humedad relativa de $88 \%$.

Todos los análisis estadísticos se realizaron utilizando el programa STATISTICA 7.0 (StatSoft, Inc., 2004).

\section{RESULTADOS}

Radiación fotosintéticamente activa: La radiación PAR varía significativamente entre épocas (MW-U $(\mathrm{N}=312) \mathrm{Z}=3.57, \mathrm{P}<0.05)$, y fue altamente fluctuante en la cima, donde los valores máximos alcanzaron los $3000 \mu \mathrm{mol} /$ $\mathrm{m}^{2}$.s en época seca, el doble de los valores registrados para la época húmeda. En general, la radiación PAR supera los $2000 \mu \mathrm{mol} / \mathrm{m}^{2}$.s en las demás zonas.

Pérdida de pubescencia foliar (PPF): La PPF en E. paipana es una enfermedad que conlleva a la formación de manchas necróticas irregulares, clorosis y la deformación de las hojas. En el haz se observó la marchitez y desprendimiento del tejido intervenal, y en el envés se evidenciaron lesiones cafés sobre la nervadura central a manera de un "raspado" que asciende hacia el ápice de la hoja causando marchitez. La incidencia de la PPF varió entre zonas $(\mathrm{KW}-\mathrm{H}(2.230)=78.68, \mathrm{P}<0.05)$ 
con mayor repercusión en los individuos de la depresión, donde el porcentaje de hojas afectadas se mantuvo entre un 37 a $55 \%$. En cada zona, el porcentaje de incidencia fue constante a lo largo de los nueve meses de seguimiento y presentó una correlación negativa con la incidencia de la herbivoría por plagas $(r=-0.62$, $\mathrm{P}<0.05)$, la cual sufrió un descenso marcado a partir de junio cuando inició la época de lluvias (Fig. 1).

Aunque la incidencia de la PPF fue constante, la severidad de la misma aumentó en promedio un $0.13 \%$ por mes, y difirió entre zonas (F (2 420) $=16.76, \mathrm{P}<0.05)$; esta fue mayor en la cima. El porcentaje de área afectada por la PPF fue mayor en el haz de las hojas $(1.420)=5.79, \mathrm{P}=0.01)$. Entre los meses de Mayo a junio se observó un rápido incremento de la severidad $(0.33 \%)$ en todas las zonas, que finalizó en julio, causando la muerte del $64.8 \%$ de las hojas con porcentajes mayores al $50 \%$ (Fig. 1). En general, el porcentaje de biomasa foliar total con PPF fue bajo en el 50 $\%$ de la población, medio en un $42 \%$ y alto en el $8 \%$ restante.

Efecto de la PPF en la fisiología de $E$. paipana: La PPF afectó significativamente la Gs $(\mathrm{F}(1.804)=315.57, \mathrm{P}<0.05)$. El intercambio gaseoso de las hojas sanas (143.2 $\mathrm{mmolH}_{2} \mathrm{O} / \mathrm{m}^{2}$.s) duplicó en promedio al de las hojas con la enfermedad $\left(68.6 \mathrm{mmolH}_{2} \mathrm{O} /\right.$ $\left.\mathrm{m}^{2} . \mathrm{s}\right)$, principalmente durante la época seca. La respuesta fue distinta para los individuos de la pendiente $(\mathrm{F}(2.804)=8.10, \mathrm{P}<0.05)$, los cuales mantuvieron valores de Gs similares durante las dos épocas, alrededor de 102.6 $\mathrm{mmolH}_{2} \mathrm{O} / \mathrm{m}^{2}$.s (Fig. 2). La Gs promedio fue mayor en los individuos de la depresión ( $\mathrm{F}$ $(2.197)=7.66, \mathrm{P}<0.05)($ Cuadro 1$)$.

Se encontró una alta variabilidad del contenido de clorofila entre hojas, por lo cual no se evidenció un patrón definido relacionado con la PPF. Con relación a la zona, el contenido de clorofila fue significativamente mayor en la pendiente $(\mathrm{F}(2.309)=11.42, \mathrm{P}<0.05)($ Fig. 2$)$.

Efecto de la época y la zona en la fisiología de $E$. paipana: Las características morfofisiológicas de los individuos de E. paipana varían entre zonas. A medida que se desciende hacia la depresión, los individuos poseen tallas más pequeñas con hojas más grandes ( $\mathrm{F}$ (2.97) $=14.75, \mathrm{P}<0.05)$, que sin importar la época logran mantener temperaturas cercanas a 19 ${ }^{\circ} \mathrm{C}$, aproximadamente $4{ }^{\circ} \mathrm{C}$ por encima de las temperaturas registradas en la cima y la pendiente $(\mathrm{F}(2.197)=35.21, \mathrm{P}<0.05)($ Cuadro 1). En contraste, durante la época seca, los individuos de la cima y la pendiente mostraron temperaturas foliares que superaron en unos 4 ${ }^{\circ} \mathrm{C}$ a las registradas para la época húmeda (Fig. 2). Al analizar las correlaciones entre variables morfo-fisiológicas, se encontró una relación

CUADRO 1

Variaciones morfo-fisiológicas de E. paipana entre zonas topográficas

TABLE 1

Morpho-physiological variations of E. Paipana among topographic zones

\begin{tabular}{lccc}
\multicolumn{1}{c}{ Características } & \multicolumn{3}{c}{ Zona } \\
morfo-fisiológicas & Cima & Pendiente & Depresión \\
Gs $\left(\mathrm{mmolH}_{2} \mathrm{O} / \mathrm{m}^{2} . \mathrm{s}\right)$ & $97.05 \pm 73.46$ & $102.63 \pm 70.27$ & $114.84 \pm 79.77$ \\
Tfol $\left({ }^{\circ} \mathrm{C}\right)$ & $17.47 \pm 3.62$ & $17.69 \pm 3.47$ & $18.23 \pm 2.53$ \\
Clorofilas $(\mathrm{ICC})$ & $29.77 \pm 10.07$ & $36.18 \pm 10.02$ & $29.66 \pm 9.33$ \\
Afol $\left(\mathrm{cm}^{2}\right)$ & $96.80 \pm 17.52$ & $139.65 \pm 33.30$ & $144.44 \pm 58.04$ \\
Altura $(\mathrm{cm})$ & $131.67 \pm 44.10$ & $112.40 \pm 29.21$ & $98.15 \pm 35.65$ \\
\hline
\end{tabular}

Media \pm desviación estándar, Gs: Conducticia estomática, Tfol: Temperatura foliar, Afol: área foliar. 

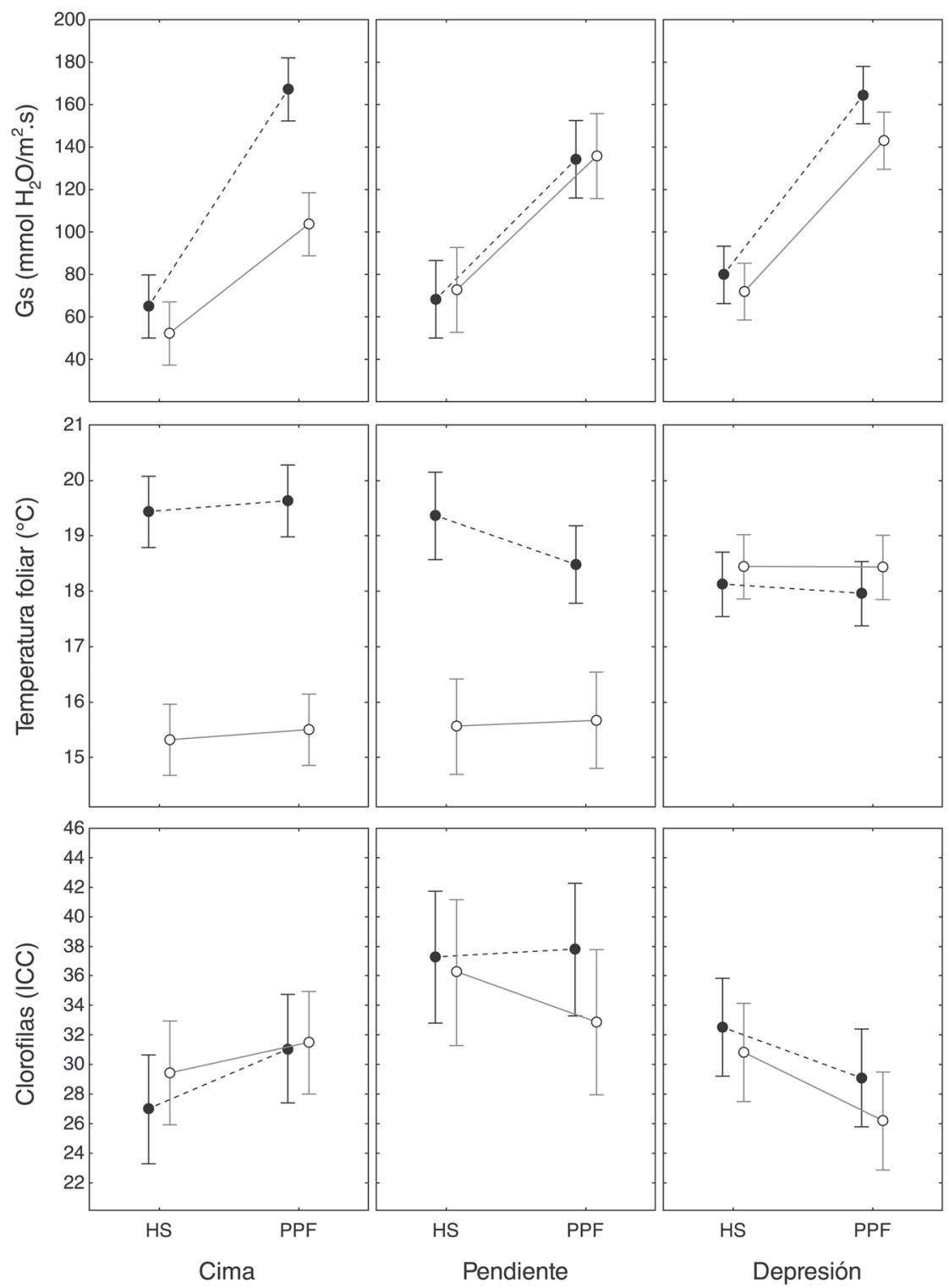

Época seca O-Época húmeda

Fig. 1. Incidencia y severidad de la PPF (Pérdida de Pubescencia Foliar) en individuos de E. paipana ubicados en cima (A), pendiente (B) y depresión (C). En la izquierda se compara la incidencia de la PPF y de la herbivoría por insectos a lo largo de nueve meses. En la derecha se muestra el avance de la severidad en el haz y el envés de hojas de durante ocho meses de seguimiento. Las franjas sombreadas indican períodos de cambios significativos en los porcentajes de incidencia y severidad.

Fig. 1. Incidence and severity of PPF (Loss of leaf pubescence) in individuals of E. paipana located in top (A), slope (B) and depression (C). On the left the PPF incidence and herbivory by insects along nine months. On the right the progress of the severity on the upper and lower sides of leaves for eight months. Shading indicates periods of significant changes in the percentages of incidence and severity. 

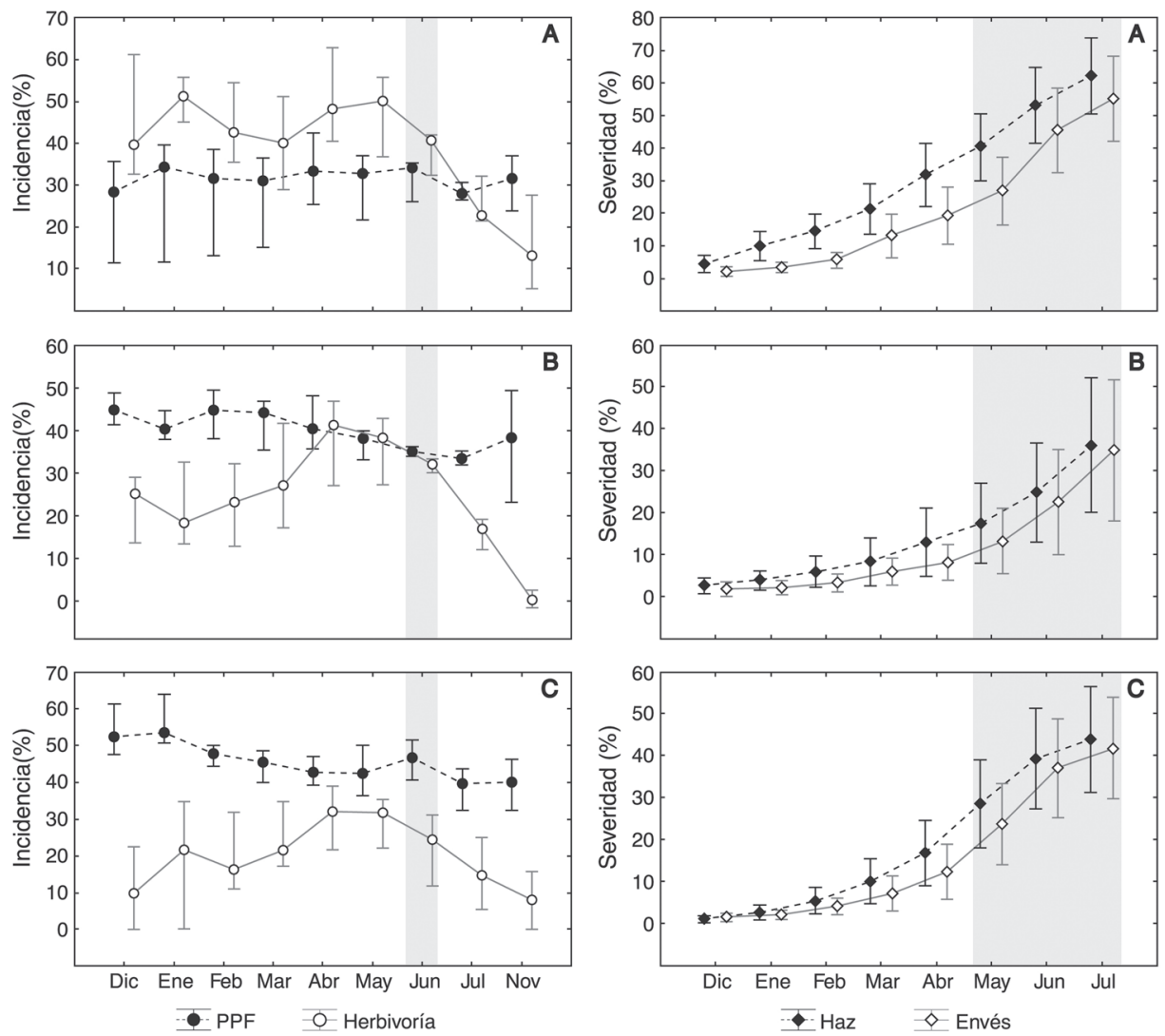

Fig. 2. Efecto de la pérdida de pubescencia foliar (PPF), zona y clima sobre las variables fisiológicas: Conductancia estomática (Gs), temperatura foliar (Tfol) e índice del contenido de clorofila (ICC). HS: Hojas sanas.

Fig. 2. Effect of the loss of leaf pubescence (PPF), zone and season on the physiological variables: Stomatal conductance (Gs), leaf temperature (Tfol) and chlorophyll content index (ICC). HS: healthy leaves.

significativa entre Gs y Tfol $(\mathrm{r}=0.44, \mathrm{P}<0.01)$ durante la época húmeda que no estuvo presente durante la época seca.

Aislamiento e identificación de hongos: A partir de las muestras del tejido foliar se aislaron 117 hongos y se logró identificar 16 géneros con una alta representación de Nigrospora (15.5\%), Botrytis (12.9\%), Cladosporium (11.25\%) y Fusarium (13.8\%), este último con dos morfotipos clasificados como cepa F1 y cepa F2. Dentro de los géneros menos frecuentes se encontró a Sarcinella (0.9 \%) y Endocochlus (1.7\%) (Cuadro 2).
Algunas cepas no presentaron cuerpos de fructificación que permitieran su identificación y por tanto fueron agrupados como micelios estériles. Botrytis, Cladosporium y Alternaria fueron los géneros más frecuentes en los individuos con PPF a partir de los cuales se obtuvieron las muestras.

En las improntas realizadas en campo, se observaron esporas y algunos residuos miceliares de hongos de los géneros Nigrospora, Epicoccum, Brachysporium, Alternaria, Fusicladium, Cladosporium, Fusarium, Spegazzinia, Sporormiella, Torula y Chaetomium. A partir de los ensayos tanto en campo como en 
CUADRO 2

Hongos asociados a la pérdida de pubescencia foliar (PPF) en E. paipana

TABLE 2

Fungi associated with the loss of leaf pubescence (PPF) in E. paipana

\begin{tabular}{|c|c|c|c|c|}
\hline \multirow{2}{*}{ Hongo/morfo-especie } & \multirow{2}{*}{ Hábito } & \multicolumn{3}{|c|}{ Frecuencia relativa } \\
\hline & & Asl & Imp & Ind \\
\hline Alternaria sp. Cepa A1t & Parásito/saprófito & 9.52 & 1.49 & 7.84 \\
\hline Botrytis sp. & Parásito & 12.98 & 10.44 & 12.74 \\
\hline Chaetomium & Saprófito & 2.59 & 8.90 & 3.92 \\
\hline Cladosporium & Parásito/saprófito & 11.25 & 11.94 & 10.78 \\
\hline Endocochlus & Saprófito & 1.73 & - & 2.74 \\
\hline Fusarium sp. cepa F1 & Parásito/saprófito & 9.52 & 1.49 & 6.95 \\
\hline Fusarium sp. cepa F2 & Parásito/saprófito & 4.33 & - & 2.85 \\
\hline Fusicladium & Parásito & 5.19 & 4.47 & 7.84 \\
\hline Nigrospora & Parásito/saprófito & 15.5 & 8.95 & 11.7 \\
\hline Oidiodendron & Saprófito & 4.33 & 2.98 & 4.9 \\
\hline Penicillium & Parásito/saprófito & 1.73 & - & 3.92 \\
\hline Rizoctonia & Parásito facultativo & 5.19 & - & 5.88 \\
\hline Sarcinella & Saprófito & 0.87 & - & 1.96 \\
\hline Sordaria & Parásito/saprófito & 4.33 & 8.95 & 4.9 \\
\hline Trichoderma & Saprófito & 5.19 & - & 4.9 \\
\hline Verticillium & Parásito/saprófito & 6.06 & - & 5.88 \\
\hline Alternaria sp.1 & Parásito/saprófito & - & 1.49 & - \\
\hline Alternaria $\mathrm{sp} .2$ & Parásito/saprófito & - & 5.97 & - \\
\hline Brachysporium & Saprófito & - & 14.92 & - \\
\hline Bactrodesmiella & Saprófito & - & 5.90 & - \\
\hline Capnodium & Patógeno & - & 7.46 & - \\
\hline Epicocum & Saprófito & - & 7.46 & - \\
\hline Spegazzinia & Saprófito & - & 4.47 & - \\
\hline Sporormiella & Saprófito & - & 2.98 & - \\
\hline Torula & Saprófito & - & 4.47 & - \\
\hline
\end{tabular}

Asl: Aislamientos, Imp: Improntas, Ind: Individuos de E. paipana.

laboratorio, se observaron tres morfotipos distintos para el género Alternaria, de los cuales se obtuvo la cepa Alt1 (Cuadro 2).

Pruebas de patogenicidad: Por su fácil aislamiento, purificación y replicación, se seleccionaron los hongos Botrytis sp, Fusarium sp. cepa F1 y Fusarium sp. cepa F2 para las pruebas de patogenicidad. A pesar de estar presentes persistentemente en las lesiones, no fue posible reproducir los síntomas observados en campo durante los 42 días de evaluación de la prueba. Sin embargo, se logró describir el comportamiento de los hongos seleccionados sobre el tejido foliar sano de E. paipana y de E. nemekenei.

Las hojas inoculadas con Botrytis sp. presentaron síntomas a los 10 días. Inicialmente se observaron manchas necróticas de color marrón distribuidas heterogéneamente sobre el envés. A los 20 días, las manchas crecieron y se unieron diseminándose desde el ápice hacia la base, dando a la hoja un aspecto blando y flexible. En algunos casos, el crecimiento del micelio sobrepasó la pubescencia de la hoja, exhibiendo cuerpos fructíferos y esclerocios de color pardo. De esta manera, a partir del día 28 las hojas comenzaron a morir con porcentajes de severidad superiores al $50 \%$ (Fig. 3).

Fusarium sp. cepa F1 causó pudrición blanda en la base de la vena principal de la hoja, tornándose de color purpura-café, y en algunos casos produjo micelio en la superficie del envés. La incidencia de este patógeno fue baja, con porcentajes de severidad menores al $30 \%$ hasta el día 28, cuando las hojas 


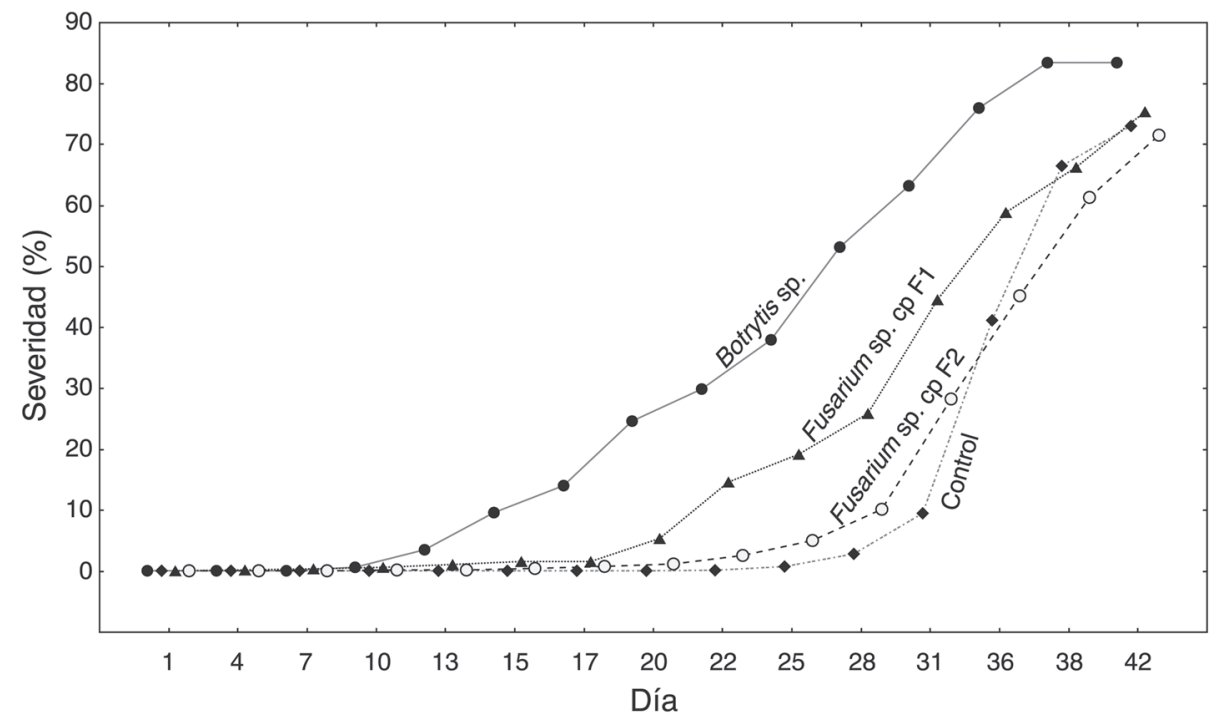

Fig. 3. Severidad de las cepas de Botrytis sp., Fusarium sp. - F1 y Fusarium sp. - F2, evaluada en hojas sanas de E. paipana y E. nemekenei durante 42 días.

Fig. 3. Severity of the strains of Botrytis sp., Fusarium sp. - F1, and Fusarium sp. - F2, evaluated in E. paipana and E. nemekenei healthy leaves during 42 days.

comenzaron a morir por pérdida de turgencia y necrosis. Por otro lado, las hojas inoculadas con Fusarium sp. cepa F2 presentaron manchas circulares negras cuyo diámetro aumentaba en el tiempo, generando grietas y marchitamiento, síntomas asociados con la invasión del hongo a través de los vasos conductores de la hoja, en este caso el porcentaje de severidad aumentó a partir del día 31, causando la muerte de la mayoría de las hojas (Fig. 3).

\section{DISCUSIÓN}

Aunque la incidencia de la PPF en $E$. paipana tiende a ser constante en el tiempo, la severidad aumenta de manera intensiva iniciando con un porcentaje de área foliar afectada menor al $10 \%$ y llegando al $45 \%$ en la depresión y al $65 \%$ en la cima al final de ocho meses de seguimiento. El efecto foto-oxidativo de la radiación diurna puede ser considerado un factor que intensifica la severidad de la enfermedad en el haz de las hojas, debido a que diariamente están expuestas a valores PAR superiores a $2000 \mu \mathrm{mol} / \mathrm{m}^{2}$.s. Esta radiación sobrepasa ampliamente los umbrales de fotoinhibición para la gran mayoría de las plantas, incluso para aquellas consideradas heliófitas (1445.16 $\mu \mathrm{mol} / \mathrm{m}^{2} . \mathrm{s}$, Fitter \& Hay, 2002). El avance de la severidad de la PPF fue estimado en alrededor de $7.68 \mathrm{~cm}^{2}$ de área foliar/mes. Esta tasa de incremento fue similar entre zonas y conllevó a la pérdida de un importante número de hojas en toda la población.

La correlación negativa entre la incidencia de la herbivoría por plagas y la incidencia de la $\mathrm{PPF}$, sugiere que existe una relación antagónica entre los agentes causantes de estos dos tipos de daño foliar. La herbivoría afecta en mayor proporción a los individuos ubicados en la cima, principalmente durante las épocas secas del año. Cuando el área foliar se reduce a causa del consumo por insectos, disminuye el transporte de agua y nutrientes en la planta, haciéndolas menos tolerantes a la depredación y más susceptibles al estrés hídrico (Tyree, Velez, \& Dalling, 1998; Cavender \& Bazzaz, 2000). Sin embargo, al iniciar la temporada de lluvias en el mes de junio la herbivoría se reduce y la PPF permanece constante. 
La reducción del área foliar funcional, a causa de la PPF, limita significativamente la eficiencia de los procesos fisiológicos en $E$. paipana. En hojas sanas, la pubescencia abundante aumenta la capa límite, reduciendo el choque térmico con el ambiente y permitiendo la apertura estomática y la difusión de gases durante el día (Meinzer \& Golstein, 1985; Gates, 1980). Se ha visto que con el incremento de la altitud, la pubescencia foliar sirve para asegurar el desacople térmico de la temperatura ambiente y reflejar un mayor porcentaje de radiación, así esto implique una menor fijación de carbono (Sandquist \& Ehleringer, 1997).

La pubescencia es considerada la primera barrera frente a la alta radiación fotosintéticamente activa y UV-B. Llegando a reflectar más del $20 \%$ de la radiación incidente, con el fin de evitar el daño en el fotosistema II y la degradación de otros pigmentos (Lang \& Schindler, 1994; Molina-Montenegro, 2008; Ranjbarfordoei, Samson, \& Van Damme, 2011). Aunque no se comprobó un efecto negativo en los contenidos de clorofilas asociado a la PPF, se observó que las hojas afectadas presentan signos de fotoinhibición, evidentes por una reducción de la media de conductancia estomática de alrededor del $48 \%$ durante la franja del mediodía. Esto conlleva a bajas tasas de asimilación de $\mathrm{CO}_{2}$ (Goldstein, et al., 1989; Ázocar \& Rada, 1993; Castrillo, 2006) que a su vez afectarán el ritmo de crecimiento y la reposición de biomasa.

E. paipana presenta un promedio de conductancia estomática $\left(115.27 \mathrm{mmolH}_{2} \mathrm{O} / \mathrm{m}^{2} . \mathrm{s}\right)$ menor al que se ha medido en Espeletias típicas de los páramos venezolanos como $E$. schultzii $\left(150\right.$ y $\left.100 \mathrm{mmolH}_{2} \mathrm{O} / \mathrm{m}^{2} . \mathrm{s}\right)$ y $E$. neriifolia $\left(200-250 \mathrm{mmolH}_{2} \mathrm{O} / \mathrm{m}^{2} . \mathrm{s}\right)$ (Cavieres, Rada, Azócar, García-Núñez, \& Cabrera, 2000; Azócar \& Rada, 2006). Para poblaciones de $E$. schultzii se ha demostrado que la Gs se reduce en un $34 \%$ al ascender de 2800 a $4200 \mathrm{~m}$ (Rada, Azócar, González, \& Briseño, 1998), mientras que con E. neriifolia se ha establecido que la asimilación neta de $\mathrm{CO}_{2}$ durante días nublados se reduce a valores críticos, cercanos al punto de compensación (Cavieres, et al.,
2000). Estas evidencias son solo una muestra de la complejidad de las respuestas funcionales de las rosetas suculentas frente a la variabilidad ambiental de alta montaña, aunque en general sugieren que las espeletias son plantas que apuestan a rendimientos fisiológicos bajos pero seguros, gracias a características morfoanatómicas de alta adaptación al páramo.

La zona topográfica donde están ubicados los individuos de E. paipana es un factor decisivo en la morfo-fisiología de la especie, de igual o mayor importancia que la PPF. El contenido de clorofila fue en promedio mucho mayor en los individuos de la pendiente en comparación con la depresión y la cima, lo que puede indicar que existe una importante influencia del ángulo de orientación con relación a la irradiación. Los individuos de la cima y la depresión, al estar expuestos de forma perpendicular a la radiación solar, producirían una menor cantidad de pigmentos fotosintéticos y harían una mayor inversión en pigmentos fotoprotectores (Manuel, et al., 1999; Körner, 2003; Molina-Montenegro \& Cavieres, 2010).

En la cima hubo una correlación altamente positiva entre la Tfol y la Gs durante la época húmeda, que sugiere que el desacoplamiento con el exterior se reduce significativamente por efecto de una mayor influencia directa del viento (Kozlowski \& Pallardy, 1979; Allen \& Ort, 2001), llevando a que la planta tenga una menor capacidad de regulación térmica y mayores limitaciones difusivas para hacer fotosíntesis. Estas plantas también presentan una menor área foliar para reducir la fotoinhibición por la alta radiación que se presenta en la cima durante la época seca (Valladares, et al., 2002; Colmenares, Rada, \& Luque, 2005).

Por otra parte, en la depresión los individuos poseen hojas más grandes que en promedio mantienen una mayor temperatura, con valores cercanos a los óptimos para la fotosíntesis en E. neriifolia a $3200 \mathrm{~m}\left(19.4{ }^{\circ} \mathrm{C}\right.$, Cavieres, et al., 2000). Teniendo en cuenta que en Espeletias los óptimos fisiológicos se reducen a medida que se asciende en altitud (Cavieres, et al., 2000; Azocar \& Rada, 2006), puede afirmarse que a $3509 \mathrm{~m}$ y con una Tfol 
media de $18.3{ }^{\circ} \mathrm{C}$, E. paipana podría realizar una fotosíntesis óptima asociada a una alta Gs. La regulación de la temperatura foliar en la depresión persiste sin importar la época del año, y podría verse favorecida por la arquitectura densa y de bajo porte de la roseta, que minimiza la exposición al aire frío (Goldstein, Meinzer, \& Monasterio, 1985).

El alto nivel de afectación del $8 \%$ de la población, la pérdida de biomasa foliar, sumado a los efectos sobre el desempeño físiológico de los individuos, sugieren que la PPF es un serio causante de mortalidad en la población de E. paipana. La comunidad de hongos asociados al tejido foliar sin pubescencia, comprende en su mayoría una gran variedad de hongos endófitos, de los cuales algunos géneros como Fusarium, Botrytis y Alternaria, poseen especies con alta capacidad patogénica (Fisher \& Petrini, 1991; Agrios, 2005). Con base en la alta representatividad en los aislamientos y su poder de infección durante las pruebas de patogenicidad, la cepa Botrytis sp. es propuesta como el agente causal de la PPF.

Botrytis es un patógeno ubicuo que infecta hojas, flores y frutos, causa estrangulamiento del tallo, manchas y pudrición en tubérculos, bulbos y raíces de vegetales y frutales (Elad \& Shtienberg, 1995; Agrios, 2005). Las condiciones de alta humedad relativa, un bajo déficit de presión de vapor en la superficie de las plantas y temperaturas bajas, características de los ambientes de páramo en época húmeda, promueven la infección por Botrytis y predisponen la susceptibilidad del hospedero (Elad, 1996; Widiastuti, et al., 2011). La acción necrotrófica de este hongo le permite infectar a su hospedero a partir de heridas superficiales, donde contamina el agua extracelular con sus esporas. Posteriormente estas germinan infectando el tejido ileso, proceso que lleva a cabo mediante la producción de enzimas como la lipasa y la cutinasa (Agrios, 2005). Desde el momento en el que inicia la infección, los contenidos celulares se tornan cafés, hasta que eventualmente se produce la muerte de las partes afectadas (H.M.R., 1899).
La participación de Botrytis en conjunto con otros patógenos, pone en evidencia la existencia de mecanismos de resistencia de la planta frente al efecto negativo de la infección. Esta podría ser una de las razones por las que no se ha hecho hincapié en estudiar el estado sanitario de estas plantas y sus interacciones bióticas. Por otro lado, en este estudio se demuestra que pese a la resistencia que genéticamente (por adaptación) estas especies poseen frente a la patogenicidad de muchos microorganismos, las alteraciones drásticas en las condiciones ambientales pueden promover su vulnerabilidad a todo tipo de estrés biótico.

Aunque no fue posible reproducir los síntomas de PPF en los ensayos de patogenicidad, se sugiere que en condiciones naturales el agente causal de la infección podría actuar de manera sinérgica con otros microorganismos como Nigrospora, Cladosporium y Alternaria. Géneros comunes en lesiones foliares de hábito saprófito facultativo que pueden actuar de forma patogénica bajo condiciones de estrés (Fisher \& Petrini, 1991; Sempere \& Santamarina, 2008). Lamentablemente, en las condiciones poblacionales actuales de E. paipana, el material foliar sano es insuficiente para realizar nuevas pruebas de patogenicidad que corroboren este planteamiento.

En general, E. paipana es una especie con serios problemas que afectan su sobrevivencia y conservación. Estos incluyen las bajas posibilidades de regeneración in vitro; la reducción de la conductancia estomática que conllevarían a un bajo rendimiento fotosintético; una infección patogénica causante de la pérdida de pubescencia foliar que afecta en mayor o menor grado al $100 \%$ de la población y de severidad creciente, que a su vez limita el desempeño fisiológico de la especie. Es evidente la necesidad inmediata de una intervención in situ que podría involucrar el estudio y uso de controladores biológicos como los hongos Thrichoderma, Chaetomium y la bacteria Bacillus licheniformis (datos no mostrados), aislados de la misma especie con el fin de evitar su extinción. 


\section{AGRADECIMIENTOS}

A la Fundación Alejandro Ángel Escobar por la aceptación y financiamiento de la propuesta.

\section{RESUMEN}

Espeletia paipana es una roseta caulescente gigante en peligro de extinción, endémica del departamento de Boyacá. Con el fin de establecer si una enfermedad en la planta, atribuida a la acción patogénica de microorganismos endófitos y caracterizada por la pérdida de pubescencia foliar (PPF), puede ser causante de la acelerada mortandad poblacional, se evaluó el desempeño fisiológico de la especie con y sin PPF. La incidencia (\% de hojas afectadas por cada uno de los 27 individuos de la población actual) y severidad (\% de área foliar afectada en 135 hojas) de la PPF fueron monitoreadas durante un periodo de nueve meses, en tres zonas topográficas a diferentes altitudes. Parámetros fisiológicos como conductancia estomática (Gs) y temperatura foliar (Tfol) e índice de contenido de clorofila (ICC) fueron medidos en hojas sanas y enfermas durante cuatro días consecutivos, tanto en la época seca como en la época húmeda. El estudio fue complementado con aislamientos y pruebas de patogenicidad para identificar el agente causal de la PPF. En general, aunque la incidencia de la enfermedad en E. paipana fue constante a lo largo del tiempo, la severidad avanzó superando el $60 \%$ del área foliar. El aumento de la severidad en el haz fue atribuido al efecto foto-oxidativo de las altas radiaciones entre las 11:00 y 14:00 h. La reducción del área foliar funcional a causa de la PPF, llevó a una baja Gs con serias implicaciones en la fijación de carbono, y por tanto limitando el crecimiento y la renovación de biomasa. El efecto estacional en la Tfol varió de acuerdo con la zona topográfica, mientras que el ICC no presentó un patrón definido con relación a la PPF; sus valores bajos podrían estar relacionados con la producción de otros pigmentos. Finalmente, aunque no es posible asegurar que Botrytis sp. es el causante de la pérdida de pubescencia foliar, se postula como el agente causal más probable debido a su alta representatividad en los aislamientos y su potencial de infección durante las pruebas de patogenicidad. En general, la reducción de biomasa foliar sana y disminución del rendimiento fisiológico sugieren que la PPF afecta negativamente la sobrevivencia de E. paipana, lo que significa que el uso de biocontroladores podría ser una estrategia para el rescate poblacional.

Palabras clave: Espeletia paipana, pubescencia, enfermedad patogénica, severidad, temperatura foliar, conductancia estomática, contenido de clorofila.

\section{REFERENCIAS}

Agrios, G. N. (2005). Plant pathology. California: Editorial Elsevier Academic Press.

Allen, D., \& Ort, D. R. (2001). Impacts of chilling temperatures on photosynthesis in warm-climate plants. Illinios: Editorial Elsevier Science.

Alonso-Amelot, M. E. (2008). High altitude plants, chemistry of acclimation and adaptation. In A. Rahman (Ed.), Studies in natural products chemistry (pp. 883983). Pakistan: Editorial Elsevier Academic Press.

Azócar, A. \& Rada, F. (1993). Ecofisiología de plantas de la alta montaña andina. En A. Azocar (Ed.), Repuestas Ecofisiológicas de plantas de ecosistemas tropicales (pp. 82-110). Mérida: Ediciones del CIELAT.

Azócar, A. \& Rada, F. (2006). Ecofisiología de plantas de páramo. Mérida: Editorial Litorama.

Barnett, H. L. (1960). Illustrated genera of imperfect fungi. Minneapolis: Editorial Burguess Publishing Company.

Castaño-Zapata, J., \& Salazar, H. (1998). Illustrated guide for identification of plant pathogens. Manizales: Universidad de Caldas.

Castaño, C. (2002). Páramos y ecosistemas alto andinos de Colombia en condición hotpost \& global climatic tensor. Bogotá: Instituto de Hidrología, Meteorología y Estudios ambientales IDEAM.

Castrillo, M. (2006). Fotosíntesis en tres poblaciones altitudinales de la planta andina Espeletia schultzii (Compositae). Revista Biología Tropical, 54(4), 1143-1149.

Cavender, J., \& Bazzaz, F. (2000). Changes in drought response strategies with ontogeny in Quercus rubra: implications for scaling from seedlings to mature trees. Acta Oecologica, 124(1), 8-18.

Cavieres, L. A., Rada, F., Azocar, A., García-Núñez, C., \& Cabrera, H. M. (2000). Gas exchange and low temperature resistance in two tropical high mountain tree species from the Venezuelan Andes. Acta Oecologica, 21(3), 203-211.

Colmenares, M., Rada, F., \& Luque, R. (2005). Anatomía foliar de Polylepis sericea wedd. (Rosaceae) a dos altitudes en los Altos Andes Venezolanos. Revista Plántula, 3(3), 141-148.

Elad, Y. (1996). Mechanisms involved in the biological control of Botrytis cinerea incited diseases. European Journal of Plant Pathology, 102(8), 719-732.

Elad, Y., \& Shtienberg, D. (1995). Botrytis cinerea in greenhouse vegetables: chemical, cultural, physiological and biological controls and their integration. Integrated Pest Management Reviews, 1(1), 15-29. 
Fisher, P. J., \& Petrini, O. (1991). Fungal saprobes and pathogens as endophytes of rice (Oryza sativa L.). New Phytologist, 120(1), 137-143.

Fitter, A. H., \& Hay, R. K. M. (2002). Environmental Physiology of Plants. San Diego: Academic Press.

French, E. R., \& Hebert, T. T. (1980). Métodos de investigación fitopatológica. San José: Instituto Interamericano de Ciencias Agrícolas de la OEA.

Gates, D. (1980). Biophysical Ecology. New York: Springer-Verlag.

Goldstein, G., Meinzer, F., \& Monasterio, M. (1985). Physiological and mechanical factors in relation to size-dependent mortality in an Andean giant rosette species. Acta Oecológica, 6(20), 263-275.

Goldstein, G., Rada, F., Canales, M. O., \& Zabala, O. (1989). Leaf gas exchange of two giant caulescent rosette species. Acta Oecologica (Oecologia Plantarum), 10(4), 359-370.

H.M.R. (1899). Botrytis and its host. The American Naturalist, 33(393), 753-754.

Hanlin, R. (1990). Illustrated genera of ascomicetes. Minnesota: APS Press.

Hofstede, R. (1995). The effects of grazing and burning on soil and plant nutrient concentrations in Colombian Páramo Grasslands. Plant and Soil, 173(1), 111-132.

Hofstede, R., Segarra, P., \& Mena, P. (2003). Los páramos del mundo. Proyecto atlas mundial de los páramos. Quito: Global Peatland Initiative/NC-IUCN/ EcoCiencia.

Körner, C. (2003). Alpine Plant Life: Functional Plant Ecology of Mountain Ecosystems. Berlin: Editorial Springer.

Kozlowski, T. T., \& Pallardy, G. G. (1979). Effects of low temperature on leaf diffusion resistance of Ulmus americana and Fraxinus pennsylvanica seedlings. Canadian Journal of Botany, 57(21), 2466-2470.

Lang, M., \& Schindler, C. (1994). The effect of leafhairs on blue and red fluorescence emission and on zeaxanthin cycle performance of Senecio medley L. Journal of Plant Physiology, 144(6), 680-685.

Levizou, E., Drilias, P., Psaras, G., \& Manetas, Y. (2005). Nondestructive assessment of leaf chemistry and physiology through spectral reflectance measurements may be misleading when changes in trichome density co-occur. New Phytologist, 165(2), 463-472.

López, F. P. (2004). Diagnóstico del estado de conservación de "Espeletia paipana" (Tesis inédita de pregrado). Universidad Pedagógica y Tecnológica de Colombia, Tunja, Colombia.

Luteyn, J. L. (1992). Páramos: why study them?. In H. Balslev, \& J. L. Luteyn (Eds.), Páramo: An andean ecosystem under human influence (pp. 1-14). London: Academic Press.
Luteyn, J. L. (1999). Páramos: A Checklist of plant diversity, Geographic Distribution and Botanical Literature. New York: Memoris of the New York Botanical Garden.

Manuel, N., Cornic, G., Aubert, S., Choler, P., Bligny, R., \& Heber, U. (1999). Protection against photoinhibition in the alpine plant Geum montanum. Oecologia, 119(2), 149-158.

Meinzer, F., \& Goldstein, G. (1985). Some Consequences of leaf pubescence in the Andean Giant rosette plant Espeletia timotensis. Ecology, 66(2), 512-520.

Molina-Montenegro, M. A. (2008). Variación de la pubescencia foliar en plantas y sus implicaciones funcionales a lo largo de gradientes altitudinales. Ecosistemas, 17(1), 146-154.

Molina-Montenegro, M. A., \& Cavieres, L. A. (2010). Altitudinal variation of morpho-physiological traits in two High-Andean plant species and its effects against the photoinhibition. Gayana. Botánica, 67(1), 1-11.

Monasterio, M. (1980). Estudios ecológicos en los Páramos Andinos. Mérida: Universidad de Los Andes.

Monasterio, M., \& Sarmiento, L. (1991). Adaptive radiation of Espeletia in the cold andean tropics. Trends Ecology and Evolution, 6(12), 387-391.

Paul, A. R. (1982). Winter leaf spot on Borage caused by Nigrospora oryzae. Australasian Plant Pathology, 11(1), 9-10.

Rada, F., Azocar, A., González, J., \& Briceño, B. (1998). Leaf gas exchange in Espeletia schultzii a giant caulescent rosette plant along an altitudinal gradient in the Venezuelan Andes. Acta Oecologica, 19(1), 73-79.

Ranjbarfordoei, A., Samson, R., \& Van Damme, P. (2011). Photosynthesis performance in sweet almond [Prunus dulcis (Mill) D. Webb] exposed to supplemental UV-B radiation. Photosynthetica, 49(1), 107-111.

Sánchez, C., \& Cely, G. (2003). Evaluación del estado de conservación de la vegetación en la reserva la Ranchería, Paipa, Boyacá. (Tesis inédita de pregrado). Universidad Pedagógica y Tecnológica de Colombia. Tunja, Colombia.

Sandquist, D., \& Ehleringer, J. (1997). Intraspecific variation of leaf pubescence and drought response in Encelia farinose associated with contrasting desert environments. New Phytologist, 135(4), 635-644.

Sempere, F., \& Santamarina, M. P. (2008). Suppression of Nigrospora oryzae (Berk. \& Broome) Petch by an aggressive mycoparasite and competitor, Penicillium oxalicum Currie \& Thom. International Journal of Food Microbiology, 122(1-2), 35-43.

Solano, C., Roa, C., \& Calle, Z. (2005). Estrategia de desarrollo sostenible. Corredor de conservación Guantiva - La Rusia - Iguaque. Boyacá-Santander/Colombia. Bogotá: Fundación Natura. 
Tyree, M., Velez, V., \& Dalling, J. W. (1998). Growth dynamics of root and shoot hydraulic conductance in seedlings of five neotropical tree species: scaling to show possible adaptation to different light regimes. Oecologia, 114(3), 293-298.

Valladares, F., Chico, J. M., Aranda, I., Balaguer, L., Dizengremel, P., Manrique, E., \& Dreyer, E. (2002). The greater seedling high-light tolerance of Quercus robur over Fagus sylvatica is linked to a greater physiological plasticity. Trees, 16(6), 395-403.
Vargas, O., Jaimes, V., Castellano, L., \& Mora, J. (2004). Proyecto Páramo andino. Propuesta de actividades de investigación para los páramos de Colombia. Bogotá: Universidad Nacional de Colombia.

Widiastuti, A., Yoshino, M., Saito, H., Maejima, K., Zhou, S. Y., Odani, H., Hasegawa, M., Nitta, Y., \& Sato, T. (2011). Induction of disease resistance against Botrytis cinerea by heat shock treatment in melon (Cucumis melo L.). Physiological and Molecular Plant Pathology, 75(4), 157-162. 